\title{
THE ABDOMEN-II
}

\author{
D A Nicholson, P A Driscoll
}

Radiographic signs of trauma

\author{
Radiographic signs of splenic injury \\ Normal abdominal radiograph \\ Raised left hemidiaphragm \\ Left pleural effusion \\ Left basal atelectasis \\ classic triad \\ Left lower rib fracture \\ Left upper quadrant mass and medial \\ displacement of gastric air bubble or \\ inferomedial displacement of splenic flexure \\ Splenic enlargement-usually with \\ subcapsular haemorrhage \\ Haemoperitoneum—see text
}

Many of the disease processes that acutely affect the abdomen produce radiographic signs, but most of these features are not specific for a particular disease process or injury.

\section{Blunt injuries}

Clinical examination is notoriously difficult, and although an abdominal radiograph can be taken as a first line investigation, often other imaging methods such as ultrasonography and computed tomography are needed to assess patients with multiple injuries who are haemodynamically stable and therefore do not require urgent laparotomy.

Splenic injury - Splenic rupture is the most common serious injury associated with blunt trauma in the upper abdomen. It may result in subcapsular haemorrhage with an intact capsule or capsular disruption, which is evident by intraperitoneal bleeding and haemorrhagic shock. Blood usually accumulates in the left paracolic gutter, producing increased soft tissue density between the descending colon and the properitoneal line. A raised left hemidiaphragm should always be considered suspicious, although this can occur normally.

Hepatic injury should be suspected in patients with a fracture of the right lower rib. Concommitant splenic injury is reported in up to a quarter of cases. A fifth show no signs or symptoms because the rupture is confined by the capsule.

Intestinal injury-The small and large intestine are rarely damaged on their own. Most patients have other more obvious injuries. Rupture of the third part of duodenum is the most common site, with signs of retroperitoneal haemorrhage or air surrounding the right kidney.

\section{Penetrating injuries}

Penetrating injuries resulting from high energy transfer are often so severe that the patient's condition will not allow formal radiographic studies. In haemodynamically stable patients, abdominal radiography is valuable for localising foreign bodies and assessing associated skeletal or soft tissue damage.

Abdominal gunshot wounds are a special problem in preoperative evaluation. Renal function often needs to be assessed urgently to exclude serious renal vascular damage. This can be done by taking an abdominal radiograph five minutes after giving intravenous iodinated contrast media. 


\section{Radiographic signs of the acute abdomen}

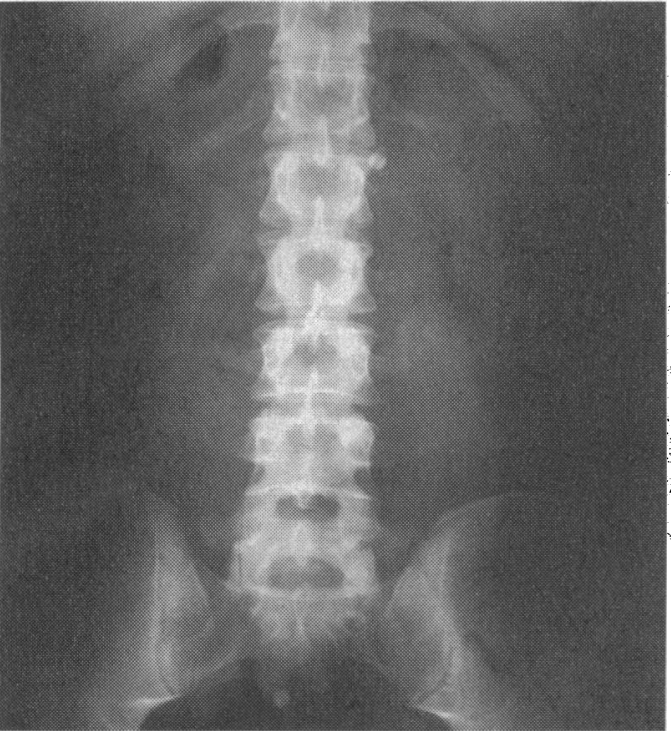

FIG 1-lsolated, dilated loop of duódenum in a patient with acute pancreatitis (sentinel loop sign).

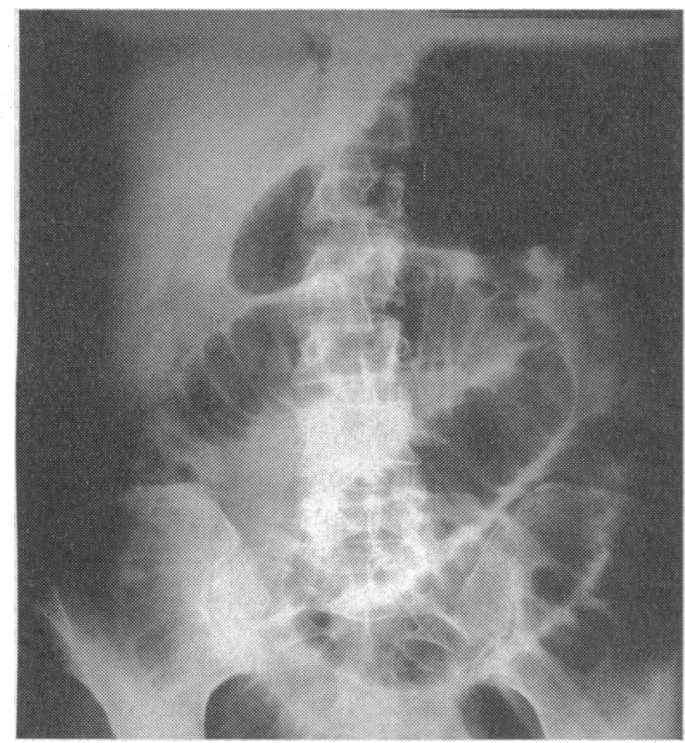

FIG 2-Complete small bowel obstruction due to surgical adhesions. The stomach and proximal small bowel are air filled and dilated. No gas is seen in the large bowel.

\section{Causes of small bowel obstruction}

Adhesions due to previous surgery (75\%)

Strangulated hernias $(10 \%)$

Appendix abscess

Gall stone ileus (2\%-commoner in women, up to $25 \%$ in elderly patients)

Crohn's disease

Tumour

Intussusception

Volvulus
Ileus

The visibility of distended loops of bowel depends on the air and fluid content. Ileus can be generalised or localised to a segment of bowel after a focal inflammatory process such as appendicitis, cholecystititis, or pancreatitis. This gives rise to the sentinal loop sign.

\section{Mechanical obstruction}

Mechanical obstruction can be partial or complete, with the distribution of distension depending on the site of obstruction.

Small bowel obstruction-Dilated small bowel loops are usually evident three to five hours after the onset of complete obstruction. They are identified by their central distribution, the presence of close valvulae conniventes that extend across the entire diameter of the bowel, and the absence of faeces. As small bowel obstruction progresses the distal bowel collapses and the colon becomes void of air. If the gut is largely fluid filled small amounts of air collect in the recesses between the valvulae conniventes, producing a chain of small radiolucent bubbles referred to as the string of pearl sign. Distended loops that are almost completely filled with fluid can be misinterpreted as ascites.

Large bowel obstruction-Dilated colon is identified by its peripheral distribution, haustral sacculations (which are thick and extend only a short distance into the gas filled lumen), and faecal content (fig 3). The distribution of bowel gas and the risk of perforation depends on the competency of the ileocaecal valve. With a competent valve the caecum is most likely to perforate as it is the most compliant region of the large bowel and distends more rapidly and to a greater degree than the remaining colon. With an incompetent ileocaecal valve, the caecum is decompressed by air passing into the small bowel. If the obstruction is complete and long standing, the gastrointestinal tract will eventually decompensate and become atonic as in ileus.

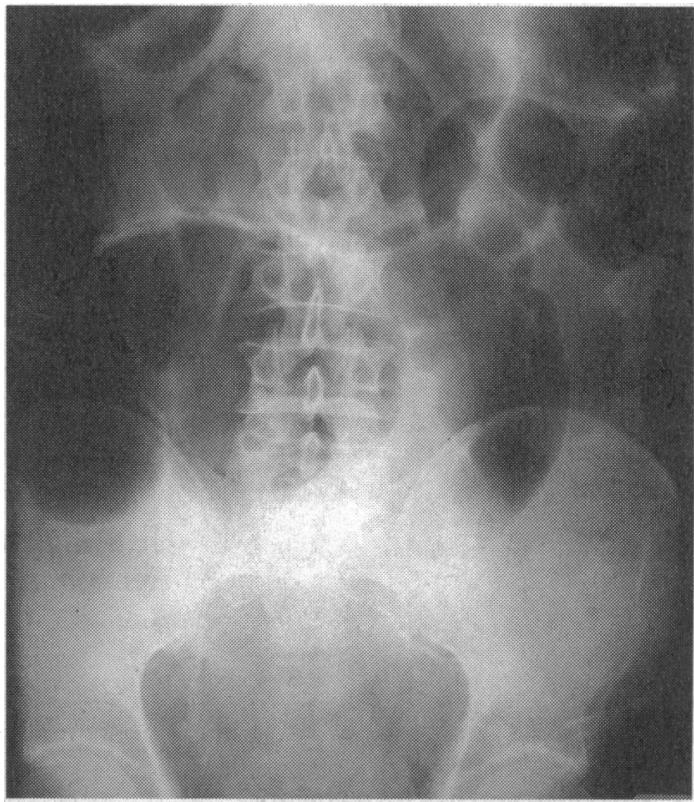

FIG 3-Large bowel obstruction due to obstructing carcinoma in the descending colon. The large bowel is dilated to the level of obstruction. No air is seen distal to this in the sigmoid colon or rectum. 


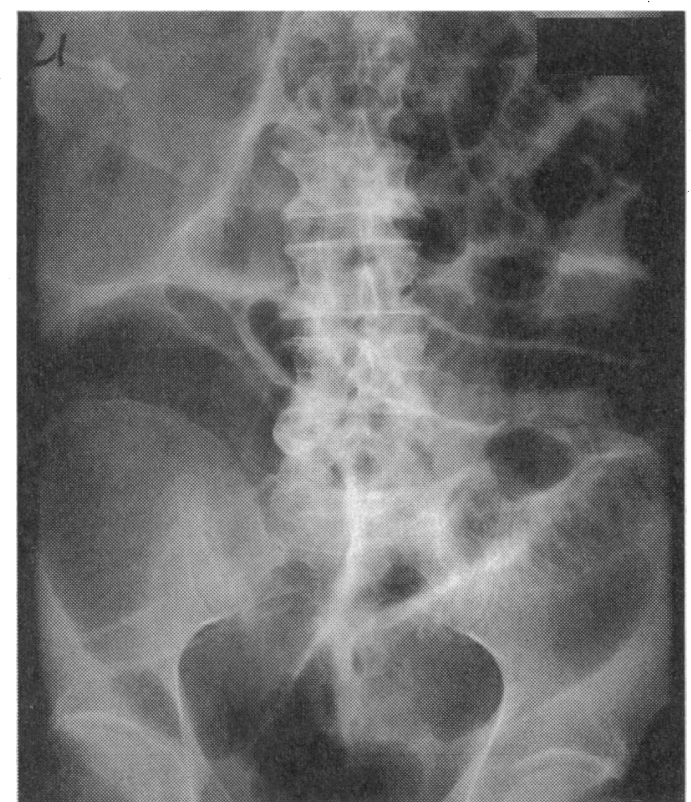

FIG 4-Pseudo-obstruction. Air is seen in the rectum and throughout the dilated small and large bowel.

The caecum is massively distended to $15 \mathrm{~cm}$ and at a high risk of perforation. No organic lesion was found.

\section{Commonest causes of large bowel obstruction}

Carcinoma (commonly sigmoid or

rectosigmoid)

Diverticular disease

Volvulus (10\%)

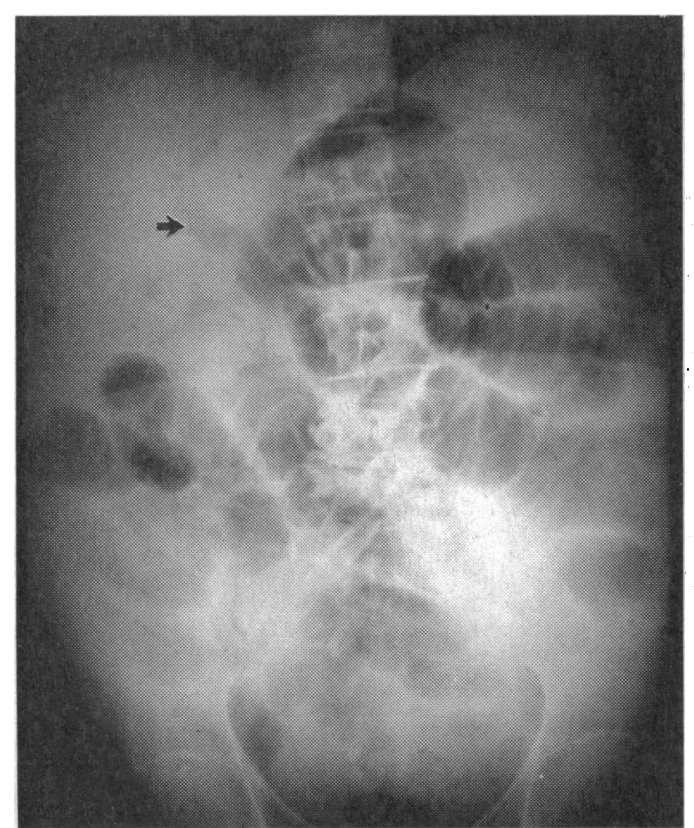

FIG 6-Gall stone ileus showing multiple, dilated; air filled loops of small bowel and gas in the biliary tree (arrow). No air is seen in the large bowel, suggesting small bowel obstruction. The gall stone is often non-opaque or obscurred and therefore not identified, as in this case.

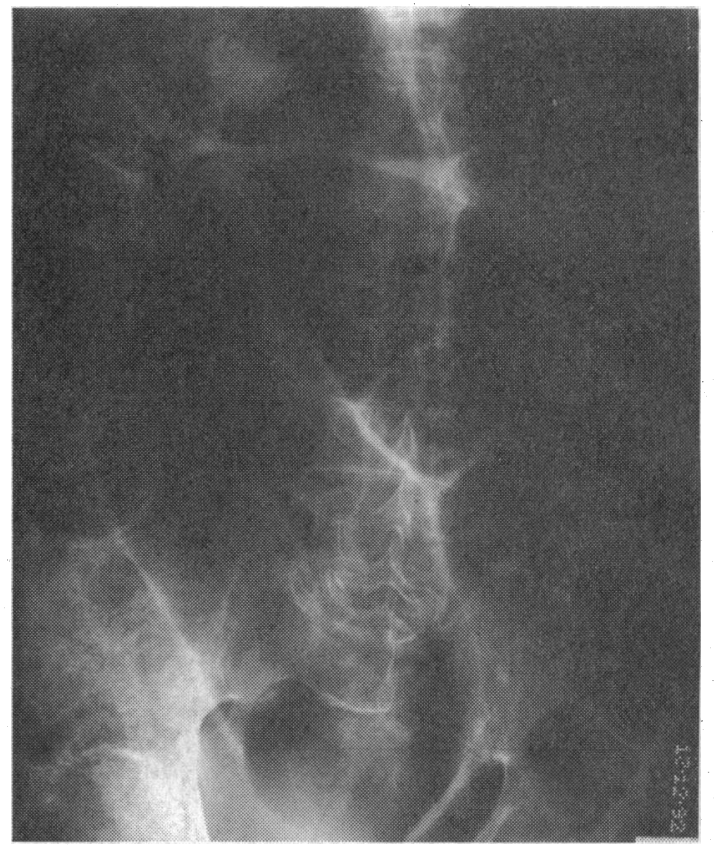

FIG 5-Classic appearance of sigmoid volvulus. The volvulus appears as a dilated, folded loop of bowel originating from the left side of the pelvis with a coffee bean configuration.

Pseudo-obstruction mimics obstruction clinically and radiologically but no obstructing lesion is found (fig 4). There is diffuse dilatation of the small and large bowel, often with prominent gastric distension. Barium enema or flexible sigmoidoscopy are often needed to differentiate pseudo-obstruction from organic obstruction.

Volvulus-Volvulus causes closed obstruction of loops, resulting in both proximal and distal portions of a loop of bowel being completely occluded. It occurs where the mesentery of the gut is longest, the most common sites being the sigmoid colon and caecum. Volvulus of the transverse colon and duodenum is rare.

In sigmoid volvulus there is a greatly dilated loop of colon, devoid of haustra, arising from the left side of the pelvis and projecting obliquely upwards to the right side of the abdomen. The volvulus overlies the distended descending colon (left flank overlap sign) and inferior border of liver (liver overlap sign). The central stripe is characteristic and is produced by the adjacent walls of the upper and lower limb of the volvulus.

Even with severe distension in caecal volvulus two haustral markings are usually identifiable. The left side of the colon is collapsed, but small bowel dilatation is often clearly seen. In half of patients with caecal volvulus the caecal pole inverts to lie in the left upper quadrant. In this orientation the wall of the dilated caecum is often kidney shaped. In the other half the twist is in the axial plane (without inversion) with the caecum remaining in the right lower quadrant.

Gall stone ileus-Gall stone ileus is the term given to mechanical obstruction caused by a gall stone that has passed into the gastrointestinal tract by eroding through the inflamed gall bladder wall (fig 6). The usual site of impaction is the pelvic or terminal ileum. Specific radiological signs are present in only $40 \%$. These include gas in the biliary tree $(30 \%)$, partial or complete small bowel obstruction (50\%), and visible ectopic gall stone $(35 \%)$. 


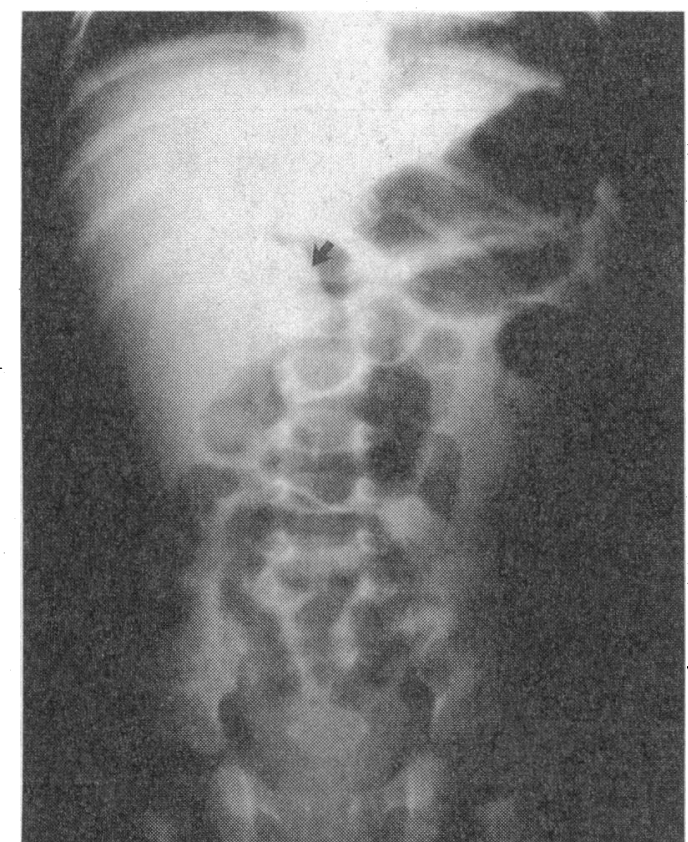

FIG 7-Intussusception in an infant. There are multiple dilated, air filled loops of bowel. The head of the ileocaecal intussusceptus can be seen in the region of the transverse colon, overlying the spine (arrow).
Intussusception is most common in children under the age of 2 years and is often due to lymphoid hyperplasia in the terminal ileum. Small bowel obstruction is seen on the abdominal radiograph possibly with a soft tissue mass surrounded by a crescent of air (fig 7).

\section{Bowel ischaemia}

The early radiological features of bowel ischaemia mimic mechanical obstruction but as the vascular occlusion progresses the bowel wall becomes oedematous and necrotic. This is seen as severe thickening of the bowel wall associated with obstruction.

Ischaemic colitis most commonly affects the splenic flexure and descending colon, with submucosal haemorrhage causing thickening of the colonic wall. These changes are seen as thumb printing (fig 8). Functional obstruction with dilatation of the proximal colon is commonly seen. As the ischaemia progresses linear gas may be identified in the bowel wall, indicating necrosis. Free gas indicates perforation, and the presence of gas in the portal vein is a serious prognostic sign.

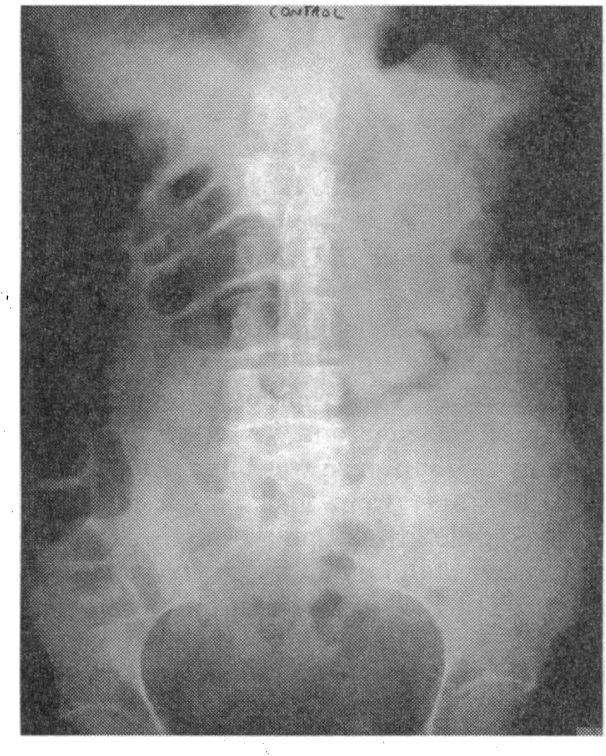

FIG 8-Ischaemic colitis of the splenic flexure with narrowing and severe submucosal

haemorrhage in the distal transverse colon (thumb printing).

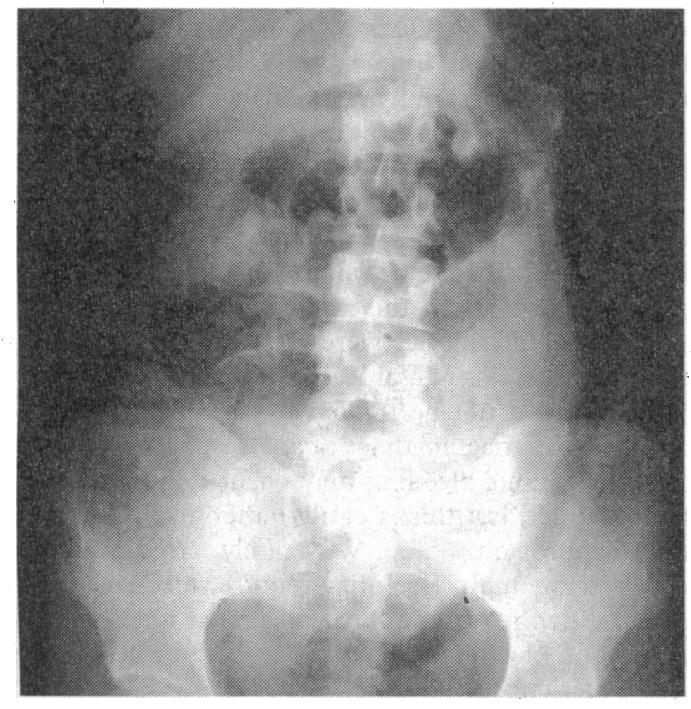

FIG 9-Acute ulcerative colitis showing oedematous left colon, which is narrowed and shortened. A cut off is seen in the transverse colon with faecal loading of the right colon due to functional obstruction.

\section{Acute inflammatory colitis}

The distribution of faecal material is a good indicator of inflammation of the bowel wall. In ulcerative colitis there is usually a sharp cut off from normal bowel, which is identified by the distal limit of the faecal residue (fig 9). With extensive mucosal ulceration small normal mucosal islands are left (pseudopolyps), which can be seen on the plain film.

Occasionally a gasless colon is seen in a patient with severe ulcerative colitis. When the transverse colonic diameter exceeds $5.5 \mathrm{~cm}$ megacolon should be diagnosed and when this is associated with fever, tachycardia, leucocytosis, and pain toxic megacolon exists. Perforation and peritonitis are common sequelae (fig 10).

\section{Acute pancreatitis}

In most patients no plain film abnormality is identified (fig 1). The signs most frequently seen, however, are a dilated duodenal sentinal loop, loss of the left psoas margin, signs of gastric outlet obstruction, and left sided pleural effusion. These features are non-specific. 


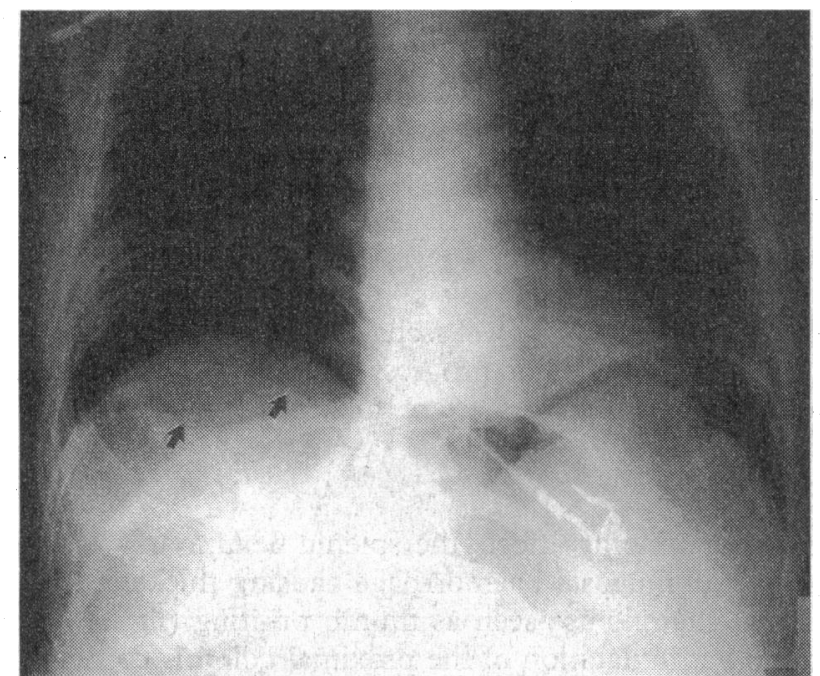

FIG 10-Erect chest radiograph showing a large pneumoperitoneum with air under both diaphragms. The right hemidiaphragm is raised. Dilated air filled loops of bowel are projected over the liver (arrows) - both sides of the bowel wall can be seen (Rigler's sign).

Summary
Diagnostic quality
Alignment of bones
Bone margins and density
Cartilage and joints
Soft tissues
Bowel gas pattern
Pneumoperitoneum
Air in the biliary tree or portal vein
Size of organs
Fat-tissue interfaces
Abnormal calcification

D A Nicholson is consultant radiologist and P A Driscoll is senior lecturer in emergency medicine, Hope Hospital, Salford.

The ABC of Emergency Radiology has been edited by David Nicholson and Peter Driscoll.

\section{Minimally Invasive Surgery}

\section{Treatment of urinary tract stones}

\section{JE A Wickham}

This is the third in a series of articles describing current techniques in minimal access surgery. The articles have been written to inform non-specialists of developments in this rapidly moving subject.
Division of Minimally Invasive Surgery, Combined Medical and Dental Schools of Guy's and St Thomas' Hospital, London

J E A Wickham, senior research fellow

BMF 1993;307:1414-7
Replacement of open surgery with minimally invasive techniques for treating stones in the renal tract has greatly reduced patients' morbidity and mortality and the period of hospitalisation and convalescence. Extracorporeal shockwave lithotripsy does not require anaesthesia and requires little analgesia so that treatment can be given on an outpatient basis, and there is no wound to heal. Only a small puncture site is needed for percutaneous endoscopic lithotomy, and with the advent of prophylactic antibiotics there are few complications. Of renal stones, about $85 \%$ can now be successfully treated by extracorporeal lithotripsy alone, and almost all of the stones too large or hard for lithotripsy can be treated endoscopically, with ultrasonic or electrohydraulic probes being used to fragment the stone. Stones in the upper and lower thirds of the ureter can be treated by extracorporeal lithotripsy, but stones in the middle third, which cannot normally be visualised to allow focusing of the shockwaves, usually require ureteroscopy. Nearly all bladder stones can be treated by transurethral endoscopy with an electrohydraulic probe. Only the largest renal tract stones still require open surgery.

\section{The renal stone}

Ten years ago it was usual for a patient suffering from a painful renal stone to undergo an open operation with a $25 \mathrm{~cm}$ loin incision to access the kidney. Two hours of anaesthesia were followed by 10-14 days in hospital, during which the patient would suffer considerable pain and discomfort. The complications of a major surgical intervention-bronchopneumonia, pulmonary embolus, and wound infection-were not unusual. ${ }^{\prime}$ Six weeks' convalescence were necessary before normal activities could be resumed.
PERCUTANEOUS ENDOSCOPIC LITHOTOMY

In 1979 at the Institute of Urology, London, and at the University of Mainz, Germany, it was realised that just as a percutaneous nephrostomy tube could be inserted into the kidney under radiological control so it might be possible to pass an endoscope down a tube track to visualise a calculus and, if it was small, remove it. In Germany this approach was initially restricted to the removal of stones from tracks pre-established at open surgery, ${ }^{2}$ while in London the first truly elective endoscopic nephrolithotomies were performed and reported in $1981 .^{3}$ By 1983 several hundred patients had been treated in Europe and the United States. ${ }^{47}$ The operation dramatically lessened patients' morbidity and mortality but gave results as good as those obtained by open surgery. Hospitalisation lasted two to three days, and a return to full activity was possible within a week. Analgesic requirements and postoperative complications were much reduced. ${ }^{8-10}$ The technique was refined, and larger stones of all types were successfully treated with ultrasonic or electrohydraulic disintegratory probes passed down the endoscope.

Complications have largely been those of postoperative septicaemia, presumably from the introduction of infective material into open venous channels in the kidney. When this was recognised appropriate prophylactic antibiotics were introduced, and this has become much less of a problem. Patients with coagulopathies should not be treated by this technique since considerable haemorrhage can result as in open surgery. Venous bleeding may occur down the percutaneous tract, but this is easily tamponaded by inserting a nephrostomy tube. Very rarely, arterial bleeding from a segmental vessel may require selective embolisation. Open retrieval surgery is almost unheard of for control of bleeding. ${ }^{11}$ Few other complications have been experienced. 